\title{
Texture Image Retrieval Based on Log-Gabor Features
}

\author{
Rodrigo Nava ${ }^{1}$, Boris Escalante-Ramírez ${ }^{2}$, and Gabriel Cristóbal ${ }^{3}$ \\ 1 Posgrado en Ciencia e Ingeniería de la Computación, \\ Universidad Nacional Autónoma de México, Mexico City, Mexico \\ urielrnv@uxmcc2.iimas . unam.mx \\ 2 Departamento de Procesamiento de Señales, Facultad de Ingeniería, \\ Universidad Nacional Autónoma de México, Mexico City, Mexico \\ boris@servidor.unam.mx \\ 3 Instituto de Óptica, Spanish National Research Council (CSIC), Serrano 121, \\ Madrid 28006, Spain \\ gabriel@optica.csic.es
}

\begin{abstract}
Since Daugman found out that the properties of Gabor filters match the early psychophysical features of simple receptive fields of the Human Visual System (HVS), they have been widely used to extract texture information from images for retrieval of image data. However, Gabor filters have not zero mean, which produces a non-uniform coverage of the Fourier domain. This distortion causes fairly poor pattern retrieval accuracy. To address this issue, we propose a simple yet efficient image retrieval approach based on a novel log-Gabor filter scheme. We make emphasis on the filter design to preserve the relationship with receptive fields and take advantage of their strong orientation selectivity. We provide an experimental evaluation of both Gabor and log-Gabor features using two metrics, the Kullback-Leibler $\left(D_{K L}\right)$ and the Jensen-Shannon divergence $\left(D_{J S}\right)$. The experiments with the USC-SIPI database confirm that our proposal shows better retrieval performance than the classic Gabor features.
\end{abstract}

Keywords: Gabor filters, Image retrieval, Jensen-Shannon divergence, Log-Gabor filters, Texture analysis.

\section{Introduction}

Due to the massive amount of digital image collections, visual information retrieval has become an active research area. The content-based image retrieval approach (CBIR) is based on extracting the content of visual information such as color [1] or textures 2] and its goal is to retrieve images from a data bank using features that best describe objects in a query image [3. Image characterization by feature extraction is used to catch similarities among images. Hence, it is a crucial stage in CBIR. Theoretically, having more features implies a greater ability to discriminate images. However, this is not always true, because not all features are important for understanding or representing a visual scene [4]. 
Texture is one of the most important features in image retrieval [5], 6]. It provides a robust mathematical description of the spatial distribution of gray levels within a bounded neighborhood and refers to visual patterns that have properties of homogeneity 7 . However, texture characterization is not an easy problem because some spatial patterns can be quite simple as stripes while others can exhibit complex behavior like those in natural images. From a mathematical point of view, it is usual to analyze the spatial distributions as intensity variations from deterministic - where textures contain periodic patterns- to randomness where textures look like unstructured noise. Since texture is a fundamental image property that describes a perceptually homogeneous region, the HVS requires that textures can be extracted and processed in an optimal way.

Spectral methods for characterizing textures have proven to be powerful tools [8. These methods collect a distribution of filter responses and extract features from the first and second order statistics [9]. Especially, the use of Gabor filters in texture analysis was motivated due to the studies of Daugman on visual modeling of simple cells. He found out that the experimental findings on orientation selectivity of visual cortical neurons were previously observed by Hubel and Wiesel in human beings and cats [10], [11, [12. Gabor filters represent timevarying signals in terms of functions that are localized in both time and frequency domains. These functions described by the product of a Gaussian function and a sinusoid constitute a unique family of linear filters that behave optimally in the sense that their simultaneous resolution in both domains is maximal [13.

Manjunath and Ma in [14] proposed a method for texture analysis. The input images are filtered using a set of Gabor filters and the mean and standard deviation are taken to build a feature vector. Their method is generally accepted as a benchmark method for texture retrieval. However, Gabor filters have not zero mean, which produces a non-uniform coverage of the Fourier domain. This distortion may cause fairly poor pattern retrieval accuracy [15].

In this paper, we propose a simple yet efficient image retrieval approach based on a novel log-Gabor filter scheme. In Section 2, the classic Gabor filter and the $\log$-Gabor model proposal are presented. In Section 3 the $D_{K L}$ and $D_{J S}$ are described. In Section 4, we compare retrieval accuracy of both Gabor and log-Gabor filter banks over the USC-SIPI database [16. Finally, our work is summarized in Section 5

\section{Bio-Inspired Models for Texture Feature Extraction}

Daugman [11 proposed a 2D extension of the Gabor filters -receptive fields are deployed in two dimensions- and showed that they occupy an irreducible volume in the four-dimensional (4D) hyperspace where the four orthogonal axes correspond to spatial $(x, y)$ and frequency $(u, v)$ variables. The joint $2 \mathrm{D}$ resolution achieves the lower bound of the $2 \mathrm{D}$ uncertainty principle as follows: $(\Delta x)(\Delta y)(\Delta u)(\Delta v) \geq \frac{1}{16 \pi^{2}}$.

The canonical 2D Gabor filter in spatial domain is defined as:

$$
g(x, y)=e^{-\frac{1}{2}\left(\frac{\left(x-x_{0}\right)^{2}+\gamma^{2}\left(y-y_{0}\right)^{2}}{\alpha^{2}}\right)+i\left(2 \pi\left[u_{0}\left(x-x_{0}\right)+v_{0}\left(y-y_{0}\right)\right]+\phi\right)}
$$


where $\left(x_{0}, y_{0}\right)$ are the center of the filter, $\left(u_{0}, v_{0}\right)$ and $\phi$ represent the radial frequency and the phase of the sinusoidal signal respectively. $(\alpha, \gamma)$ are the space constants of the Gaussian envelope along $x$ and $y$ axes respectively and they control the filter bandwidth.

Here, we assume the use of real Gabor filters (just the even part) centered at the origin. Therefore, we obtain the next expression that provides a suitable symmetric filter for detecting salient edges [17] as follows:

$$
g(x, y)=e^{-\frac{1}{2}\left(\frac{x^{2}+\gamma^{2} y^{2}}{\alpha^{2}}\right)} \cos \left(2 \pi u_{0} x\right)
$$

Using the rotation matrix, $R_{\theta}=[\cos \theta,-\sin \theta ; \sin \theta, \cos \theta]$ and applying in Eq. 2 yields the 2D polar Gabor representation as follows:

$$
g(x, y)=e^{-\frac{1}{2}\left(\frac{\tilde{x}^{2}+\gamma^{2} \tilde{y}^{2}}{\alpha^{2}}\right)} \cos \left(2 \pi u_{0} \tilde{x}\right)
$$

with

$$
\begin{aligned}
& \tilde{x}=x \cos \theta-y \sin \theta \\
& \tilde{y}=x \sin \theta+y \cos \theta
\end{aligned}
$$

The frequency and orientation selectivity properties of Gabor filters can be more explicit in Fourier domain. The Fourier transform of $g(x, y)$ is given by:

$$
\begin{aligned}
\hat{G}(u, v) & =e^{-2 \pi^{2} \alpha^{2}\left[\left(\tilde{u}-u_{0} \cos \theta\right)^{2}+\frac{1}{\gamma^{2}}\left(\tilde{v}+u_{0} \sin \theta\right)^{2}\right]} \\
& +e^{-2 \pi^{2} \alpha^{2}\left[\left(\tilde{u}+u_{0} \cos \theta\right)^{2}+\frac{1}{\gamma^{2}}\left(\tilde{v}-u_{0} \sin \theta\right)^{2}\right]}
\end{aligned}
$$

where $(\tilde{u}, \tilde{v})=(u \cos \theta+v \sin \theta,-u \sin \theta+v \cos \theta)$.

$\hat{G}(u, v)$ represents a rotated Gaussian function by an angle $\theta$ with $u_{0}$ frequency units shifted along the axes.

Psychophysical experiments showed that frequency bandwidths of simple cells are about one octave apart [11, [18, [19]. The half-amplitude bandwidth of the frequency response, $B_{u}$, satisfies this condition and is linked to central frequency $u_{0}$ as follows:

$$
\alpha=\frac{\sqrt{\log (2)}\left(2^{B_{u}}+1\right)}{\sqrt{2} \pi u\left(2^{B_{u}}-1\right)}
$$

In order to determine the optimum angular bandwidth $B_{\theta}$ we considered an isotropic Gabor filter. Hence, we forced $\gamma=1$.

$$
\frac{\alpha}{\gamma}=\frac{\sqrt{\log (2)}}{\sqrt{2} \pi u \tan \left(\frac{B_{\theta}}{2}\right)}
$$

in this way, $B_{\theta} \approx 36^{\circ}$ is obtained, but for computational efficiency $B_{\theta}=\frac{\pi}{6}$ was chosen.

Although Gabor filters possess a number of interesting mathematical properties (they have a smooth and indefinitely differentiable shape and they do not have side lobes neither in space nor frequency domain) they present a main drawback, the filter averaging is not null and therefore the DC component influences intermediate bands. They overlap more at lower frequencies than in higher ones yielding a non-uniform coverage of the Fourier domain, (see Fig. 1(a)). 


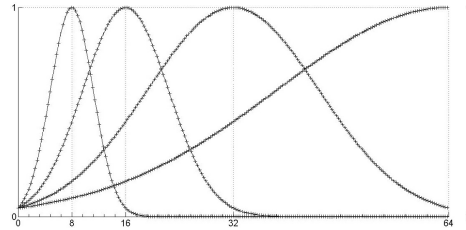

(a)

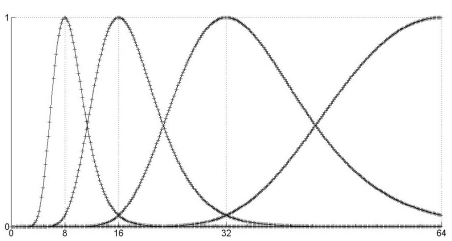

(b)

Fig. 1. Profiles of the frequency response of (a) Gabor and (b) log-Gabor filters. Note that the DC component is minimized by introducing the ln function.

\section{$2.1 \quad$ Log-Gabor Filters}

Log-Gabor filters, firstly proposed by D. Field [20], are defined in the frequency domain as Gaussian functions shifted from the origin due to the singularity of the log function. They always have a null DC component and can be optimized to produce filters with minimal spatial extent in an octave scale multiresolution scheme, (see Fig. 1(b)]. Log-Gabor filters can be splited into two components: radial and angular filters, $\hat{G}(\rho, \theta)=\hat{G}_{\rho} \hat{G}_{\theta}$, as follows:

$$
\hat{G}(\rho, \theta)=e^{-\frac{1}{2}\left[\frac{\log \left(\frac{\rho}{u_{0}}\right)}{\log \left(\frac{\alpha_{\rho}}{u_{0}}\right)}\right]^{2}} e^{-\frac{1}{2}\left[\frac{\left(\theta-\theta_{0}\right)}{\alpha_{\theta}}\right]^{2}}
$$

where $(\rho, \theta)$ represent the polar coordinates, $u_{0}$ is the central frequency, $\theta_{0}$ is the orientation angle. $\alpha_{\rho}$ and $\alpha_{\theta}$ determine the scale and the angular bandwidth respectively. We set $\alpha_{\rho}=0.75$ that results in minimal overlap among scales one octave apart and $\alpha_{\text {theta }}=\frac{p i}{6}$ as it was mentioned before. In order to better cover the Fourier plane even scales are rotated by a constant factor consisting of the half a distance between filter centers, (see Fig. 2(c)], 21].

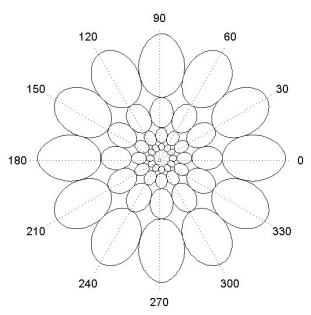

(a)

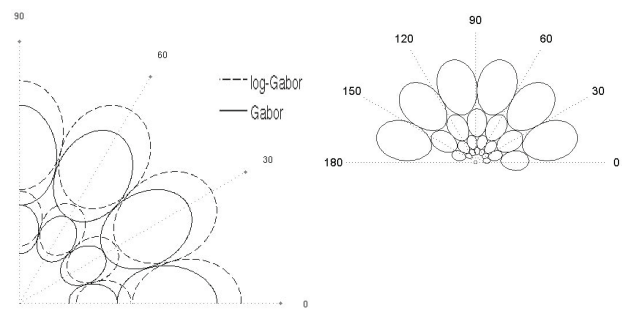

(b)

Fig. 2. Half-amplitude bandwidth of the frequency response of (a) an ensemble of Gabor filters. (b) Contour comparison between Gabor and log-Gabor filters before rotating the log-Gabor even bands. (c) Log-Gabor filters (1 octave bandwidth). 


\section{Texture Retrieval Based on Entropy Information}

As in 14], any image coefficient, $C_{(s, \theta)}$, defined as $C_{(s, \theta)}=I(x, y) \star g(x, y)_{(s, \theta)}$ where $I(x, y)$ is the given image, $g(x, y)_{(s, \theta)}$ is the filter at the scale $s$ and orientation $\theta$, and $\star$ indicates the convolution, represents texture characteristics in a particular scale and orientation. Thus, energy signatures such as the mean $\mu_{(s, \theta)}$ and the variance $\sigma_{(s, \theta)}^{2}$ can be used as texture features for constructing a feature vector as follows:

$$
\bar{t}=\left[\mu_{(0,0)}, \sigma_{(0,0)}^{2}, \ldots, \mu_{(s-1, \theta-1)}, \sigma_{(s-1, \theta-1)}^{2}\right]
$$

Although the Kullback-Leibler divergence-a generalization of Shannon's entropyis not a true metric rather it is a relative entropy, it can be used as a suitable descriptor for measuring distances between histograms or feature vectors. Then, the distance between two texture images $A$ and $B$ with $\overline{t_{A}}$ and $\overline{t_{B}}$ as the corresponding feature vectors is defined as:

$$
D_{K L}(A, B)=\sum_{i=0}^{b-1} \overline{t_{B}}(i) \log \left(\frac{\overline{t_{B}}(i)}{\overline{t_{A}}(i)}\right)
$$

where $b$ is the length of the feature vectors $\overline{t_{A}}$ and $\overline{t_{B}}$.

In addition, the Jensen-Shannon divergence 22 denoted by $\psi$ can be used for evaluating distance between two textures as follows:

$$
\psi=\sqrt{2 D_{J S}(A, B)}
$$

where

$$
D_{J S}(A, B)=\frac{1}{2} D_{K L}\left(A, \frac{A+B}{2}\right)+\frac{1}{2} D_{K L}\left(B, \frac{A+B}{2}\right)
$$

\section{Experimental Results}

We used the USC-SIPI texture database [16], to measure retrieval accuracy (RA) of both Gabor and log-Gabor filters. USC-SIPI consists of twenty gray-scale textures of $512 \times 512$ pixels. Each image was divided into sixteen $128 \times 128$ non-overlapping patches, thus creating a database of 320 texture images. The resulted patches were processed with a filter bank ( 4 scales and 6 orientations) in order to form 320 feature vectors of 48 bins-length each. Each feature vector is a query pattern and was used to calculate distances among the 320 textures. The distances were sorted in increasing order and the closest sixteen patches were retrieved. We must note that in [14 the mean and the standard deviation were used to form a query image. Here we use the mean and the variance because they improve the retrieval performance.

The average retrieval rate (ARR) is the standard metric for evaluating CBIR systems and is listed in Table 1 for the different texture images used in this study. ARR is calculated by the following procedure: First, each texture ( $\left.\mathrm{D}^{*}\right)$ is 
Table 1. ARR for the 20 texture images, $D^{*}$ indicates the Brodatz texture. ARR is computed using Gabor and log-Gabor filter banks and both $D_{K L}$ and $D_{J S}$ metrics.

\begin{tabular}{ccccccccc}
\hline & \multicolumn{3}{c}{ Gabor filters } & \multicolumn{3}{c}{ log-Gabor filters } \\
\cline { 2 - 9 } texture & \multicolumn{3}{c}{$D_{K L}$} & \multicolumn{3}{c}{$D_{J S}$} & \multicolumn{3}{c}{$D_{K L}$} & \multicolumn{2}{c}{$D_{J S}$} \\
\cline { 2 - 9 } \# patches & $(\%)$ & \# patches & $(\%)$ & \# patches & $(\%)$ & \# patches & $(\%)$ \\
\hline D1 & 256 & 100 & 256 & 100 & 256 & 100 & 256 & 100 \\
D3 & 186 & 72.65 & 181 & 70.70 & 177 & 69.14 & 175 & 68.35 \\
D4 & 256 & 100 & 256 & 100 & 256 & 100 & 256 & 100 \\
D5 & 220 & 85.93 & 224 & 87.5 & 227 & 88.67 & 229 & 89.45 \\
D6 & 256 & 100 & 256 & 100 & 256 & 100 & 256 & 100 \\
D9 & 231 & 90.23 & 233 & 91.01 & 254 & 99.21 & 254 & 99.21 \\
D10 & 191 & 74.60 & 194 & 75.78 & 217 & 84.76 & 221 & 86.32 \\
D11 & 256 & 100 & 256 & 100 & 256 & 100 & 256 & 100 \\
D15 & 183 & 71.48 & 179 & 69.92 & 194 & 75.78 & 187 & 73.04 \\
D20 & 256 & 100 & 256 & 100 & 256 & 100 & 256 & 100 \\
D24 & 240 & 93.75 & 238 & 92.96 & 243 & 92.92 & 242 & 94.53 \\
D26 & 256 & 100 & 256 & 100 & 256 & 100 & 256 & 100 \\
D56 & 256 & 100 & 256 & 100 & 253 & 98.82 & 256 & 100 \\
D66 & 178 & 69.53 & 178 & 69.53 & 200 & 78.12 & 202 & 78.90 \\
D93 & 231 & 90.23 & 234 & 91.40 & 242 & 94.53 & 244 & 95.31 \\
D104 & 256 & 100 & 256 & 100 & 256 & 100 & 256 & 100 \\
D105 & 136 & 53.12 & 136 & 53.12 & 205 & 80.07 & 203 & 79.29 \\
D106 & $\mathbf{1 3 4}$ & $\mathbf{5 2 . 3 4}$ & $\mathbf{1 3 6}$ & $\mathbf{5 3 . 1 2}$ & $\mathbf{1 7 3}$ & $\mathbf{6 7 . 5 7}$ & $\mathbf{1 7 7}$ & $\mathbf{6 9 . 1 4}$ \\
D109 & 163 & 63.67 & 163 & 53.67 & 227 & 88.67 & 227 & 88.67 \\
D112 & 200 & 78.12 & 198 & 77.34 & 190 & 74.21 & 191 & 74.60 \\
\hline
\end{tabular}

Table 2. FRR for Gabor and log-Gabor filters. Given a single query (patch), all the sixteen patches that belong to the same texture are retrieved.

\begin{tabular}{lcccc}
\hline & \multicolumn{2}{c}{ Gabor filters } & \multicolumn{2}{c}{ log-Gabor filters } \\
\cline { 2 - 5 } distance & \# patches & $(\%)$ & \# patches & $(\%)$ \\
\hline$D_{K L}$ & 146 & 45.62 & $\mathbf{1 6 5}$ & $\mathbf{5 1 . 5 6}$ \\
$D_{J S}$ & 147 & 45.93 & $\mathbf{1 6 7}$ & $\mathbf{5 2 . 1 8}$ \\
\hline
\end{tabular}

divided into 16 patches, from which each patch is used as a query. In the best case, one single query returns the 16 patches belonging to the same texture, and evaluating all the 16 queries return up to 256 patches from the same texture.

Note that for the Gabor scheme, the lower rate achieved was with the $D 106$ texture, the ARR was $52.34 \%$ and $53.12 \%$ using $D_{K L}$ and $D_{J S}$ respectively. On the contrary, the log-Gabor scheme achieved $67.57 \%$ and $69.14 \%$ of accuracy respectively, which represents 39 and 41 more patches classified correctly with $D_{K L}$ and $D_{J S}$ metrics respectively.

In the ideal case, given a single query, all the sixteen patches that belong to the same texture should be retrieved. An important metric that assesses this specific case is called full retrieval rate (FRR) which measures the number of query patterns fully retrieved correctly. Our proposal achieves $52.18 \%$ of query patterns fully retrieved, it means a $6.56 \%$ higher rate compare to the Gabor scheme with $45.62 \%$, (see Table 2).

An overall retrieval rate (ORR) is presented in Table 3. The Gabor scheme achieves $84.78 \%$ and $84.80 \%$ of patches retrieved correctly with $D_{K L}$ and $D_{J S}$ respectively. On the other hand, the proposal here presented achieves $89.72 \%$ 
Table 3. ORR for Gabor and log-Gabor schemes

\begin{tabular}{ccc}
\hline & Gabor filters & $\log$-Gabor filters \\
\cline { 2 - 3 } distance & $(\%)$ & $(\%)$ \\
\hline$D_{K L}$ & 84.78 & $\mathbf{8 9 . 7 2}$ \\
$D_{J S}$ & 84.80 & $\mathbf{8 9 . 8 4}$ \\
\hline
\end{tabular}

and $89.84 \%$ of patches retrieved correctly with $D_{K L}$ and $D_{J S}$ respectively. This represents an increase in the classification rate up to $4.94 \%$ using $D_{K L}$ and $5.04 \%$ using $D_{J S}$.

\section{Conclusions}

Here we presented the classic Gabor scheme for texture analysis and summarized its properties and drawbacks. Further, a novel scheme for CBIR was presented. This proposal based on log-Gabor filters has a strong correlation with the HVS. It may say that the proposal is a bio-inspired model where the parameters agreed with simple cells in the visual cortex. In addition, we evaluate the texture distances using two metrics, the well-known $D_{K L}$ and the Jensen-Shannon divergence, which boosts the retrieval process. The log-Gabor filtering approach outperforms the retrieval performance for the analyzed textures in comparison with the Gabor filters.

Acknowledgments. This work has been sponsored by the grant UNAM PAPIIT IN113611 and TEC2010-20307 from the Spanish Ministry of Science and Innovation. R. Nava gives a special thank to Consejo Nacional de Ciencia y Tecnología for the doctoral scholarship 167161.

\section{References}

1. Xing-yuan, W., Zhi-feng, C., Jiao-jiao, Y.: An effective method for color image retrieval based on texture. Computer Standards \& Interfaces 34(1), 31-35 (2012)

2. Huang, P.W., Dai, S.K.: Image retrieval by texture similarity. Pattern Recognition 36(3), 665-679 (2003)

3. Jie, Y., Qiang, Z., Liang, Z., Wuhan, C.Y.: Research on texture images retrieval based on the Gabor wavelet transform. In: International Conference on Information Engineering, ICIE 2009, vol. 1, pp. 79-82 (2009)

4. ElAlami, M.E.: A novel image retrieval model based on the most relevant features. Knowledge-Based Systems 24(1), 23-32 (2011)

5. Zhang, G., Ma, Z.M.: Texture feature extraction and description using Gabor wavelet in content-based medical image retrieval. In: International Conference on Wavelet Analysis and Pattern Recognition, ICWAPR 2007, vol. 1, pp. 169-173 (2007)

6. Turner, M.R.: Texture discrimination by Gabor functions. Biological Cybernetics 55, 71-82 (1986) 
7. Nava, R., Cristóbal, G., Escalante-Ramírez, B.: A comprehensive study of texture analysis based on local binary patterns. In: Optics, Photonics, and Digital Technologies for Multimedia Applications II 8436-1, 84360E. SPIE (2012)

8. Randen, T., Husøy, J.H.: Filtering for texture classification: A comparative study. IEEE Trans. Pattern Anal. Mach. Intell. 21, 291-310 (1999)

9. Nava, R., Escalante-Ramírez, B., Cristóbal, G.: A comparison study of Gabor and log-Gabor wavelets for texture segmentation. In: 7th International Symposium on Image and Signal Processing and Analysis (ISPA), pp. 189-194 (2011)

10. Kong, A.W.-K.: An Analysis of Gabor Detection. In: Kamel, M., Campilho, A. (eds.) ICIAR 2009. LNCS, vol. 5627, pp. 64-72. Springer, Heidelberg (2009)

11. Daugman, J.G.: Uncertainty relation for resolution in space, spatial frequency, and orientation optimized by two-dimensional visual cortical filters. J. Opt. Soc. Am. A 2, 1160-1169 (1985)

12. Hubel, D.H., Wiesel, T.N.: Brain and Visual Perception: The Story of a 25-year Collaboration. Oxford University Press, Oxford (2005)

13. Gabor, D.: Theory of communication. J. Inst. Elec. Eng. (London) 93(III), 429-457 (1946)

14. Manjunath, B.S., Ma, W.Y.: Texture features for browsing and retrieval of image data. IEEE Transactions on Pattern Analysis and Machine Intelligence 18(8), 837$842(1996)$

15. Sastry, C.S., Ravindranath, M., Pujari, A.K., Deekshatulu, B.: A modified Gabor function for content based image retrieval. Pattern Recognition Letters 28(2), 293300 (2007)

16. Brodatz, P.: USC-SIPI (2012), http://sipi.usc.edu/database/ database.php?volume=rotate (Online accessed March 1, 2012)

17. Redondo, R., Sroubek, F., Fischer, S., Cristóbal, G.: Multifocus image fusion using the log-Gabor transform and a multisize windows technique. Information $\mathrm{Fu}$ sion 10(2), 163-171 (2009)

18. Bovik, A.C., Clark, M., Geisler, W.S.: Multichannel texture analysis using localized spatial filters. IEEE Transactions on Pattern Analysis and Machine Intelligence 12, 55-73 (1990)

19. Clausi, D.A., Jernigan, M.E.: Designing Gabor filters for optimal texture separability. Pattern Recognition 33(11), 1835-1849 (2000)

20. Field, D.J.: Relations between the statistics of natural images and the response properties of cortical cells. J. Opt. Soc. Am. A 4(12), 2379-2394 (1987)

21. Gross, M., Koch, R.: Visualization of multidimensional shape and texture features in laser range data using complex-valued Gabor wavelets. IEEE Transactions on Visualization and Computer Graphics 1(1), 44-59 (1995)

22. Lin, J.: Divergence measures based on the Shannon entropy. IEEE Transactions on Information Theory 37(1), 145-151 (1991) 